\title{
Modèle de calcul du croît journalier de lipides et de protéines chez les bovins*
}

\author{
J Robelin \\ Laboratoire de la Production de Viande, INRA, Theix, 63122 Ceyrat, France
}

Summary - A mathematical model for predicting body gain composition of cattle is presented. Daily lipid, protein or tissue gains are predicted according to body weight and body weight gain, for male or female cattle of various breeds. The aim and the biological basis of this model are described.

\begin{abstract}
Introduction - La plupart des modèles de croissance décrits récemment chez les bovins (Hoon Song et Dinckel, 1978; Fox et Black, 1984) ne tiennent pas compte des différences de composition du croît entre races, et sont souvent basés sur des résultats de composition corporelle très disparates. Celui que nous présentons brièvement dans cette note, permet de calculer le croît journalier de lipides, de protéines, ou de l'un des différents tissus (muscles, squelette, tissu adipeux), selon le poids et la vitesse de croissance des animaux. II est applicable à plus de 15 types d'animaux, mâles, femelles ou bœufs, de différentes races et d'âge variable. Nous limiterons cette présentation au croît de lipides et de protéines.
\end{abstract}

Bases biologiques du modèle - La démarche qui a conduit à l'élaboration de ce modèle et les données expérimentales nombreuses sur lesquelles il a été validé, ont été présentées dans des publications antérieures (Robelin et Daenicke, 1980; Robelin, 1986). Ce modèle repose sur 2 types de relations.

Relations entre le poids vif des animaux et leur composition. II s'agit des relations allométriques observées chez des animaux en croissance, entre le poids des différents constituants chimiques $(L I P=$ lipides; $P R O T=$ protéines) ou des différents tissus, et le poids d'un ensemble de référence, qui peut être le poids vif $(P V)$, le poids vif vide $(P V V=\mathrm{PV}-$ contenu digestif) ou encore le poids délipidé ( $P V V$ -
$L I P$ ). Les 3 principales équations mises en cuvre sont les suivantes:

$\log P V V=a_{0}+a_{1} \log P V$

( $a_{0}$ et $a_{1}$ adaptés à chaque type d'animal) $\log L I P=b_{0}+b_{1} \log P V V+b_{2}(\log P V V)^{2} \quad$ (2) $\left(b_{0}, b_{1}, b_{2}\right.$ adaptés à chaque type d'animal)

$\log P R O T=-1,9409+1,0723 \log (P V V-L I P)$

(relation unique pour tous les types d'animaux).

Relation entre la vitesse de croissance et la composition du croît. L'accroissement du niveau alimentaire entraîne chez les bovins, une augmentation de la croissance pondérale globale accompagnée par un accroissement de la teneur en lipides du croît. Les animaux recevant un régime riche en énergie présentent donc une adiposité accrue, cet effet étant d'autant plus marqué que les animaux ont déjà une propention à l'adiposité : animaux de race précoce, femelles (Geay et al, 1976). On peut exprimer cela grâce à une relation exponentielle entre le gain de $L I P$ journalier $(\mathrm{d} L I P)$ et le gain de $P V$ (dPV) (Robelin, 1979; Robelin et Daenicke, 1980). Ainsi, chez des animaux de même type et de même poids recevant différents niveaux alimentaires, la quantité de LIP fixés est proportionnelle au gain de $P V$ élevé à la puissance 1,8 .

$\mathrm{d} L I P=\mathrm{d} L I P_{0} \cdot \mathrm{d} P V V^{\prime 8}$.

Le coefficient $\mathrm{d} L I P_{0}$ qui dépend du type d'animal et de son $P V$, est calculé à partir de la dérivée des éqns 1 et 2 et de la croissance pondérale de référence dont

\footnotetext{
" Communication aux IVes journées de Recherches sur l'Alimentation et la Nutrition des Herbivores de mars 1988, non publiée par erreur en 1989.
} 
nous préciserons la signification dans le paragraphe suivant. Le gain journalier de PROT (dPROT) est calculé à partir de la dérivée des éqns 1 et 3 , et de $\mathrm{d} L I P$ issu de l'éqn 4.

Paramétrage du modèle - Chaque type de bovin est représenté par un animal de référence caractérisé par sa courbe de croissance pondérale, et sa composition corporelle à 2 stades de développement sur cette courbe. Le modèle a pour but de simuler la composition du croît d'un animal dont la croissance pondérale diffère de la courbe de référence. Les paramètres de croissance pondérale et de composition corporelle caractérisant les différents types d'animaux ont été déterminés expérimentalement (Geay et al, 1987). Ce sont, le $P V$ initial et final, les 3 coefficients de la courbe de croissance de référence (éqn de Gompertz), le contenu digestif initial et final, le poids des LIP corporels initiaux et finaux. Les coefficients des éqns 1 à 4 sont calculés à partir de ces paramètres.

Fonctionnement du modèle - Les données d'entrée du modèle sont le type d'animal (race, sexe), $P V$ et $\mathrm{d} P V$. Le modèle calcule d'abord les caractéristiques de l'animal de référence de " $P V$ » défini par les éqns 1 à 3 . Ensuite, il calcule le dLIP et le $\mathrm{d} P R O T$ correspondant au $\mathrm{d} P V$ sou- haité ou observé. Ainsi, à un couple de valeurs $(P V, \mathrm{~d} P V)$, ce modèle fait correspondre un autre couple de valeurs ( $L I P$, $\mathrm{d} P R O T$ ). On peut donc l'utiliser pour simuler l'évolution du poids des protéines et des lipides chez un animal qui suit une courbe de croissance quelconque. II suffit pour cela de préciser l'état initial de l'animal, de définir l'évolution du $\mathrm{d} P V$ en fonction de l'âge ou du $P V$, et en procédant pas à pas, d'accumuler les $d L I P$ et les $\mathrm{d} P R O T$. Le tableau montre un exemple d'application de ce modèle à des bovins mâles Pie-noirs et Charolais, à croissance constante $(0,7$ ou $1,1 \mathrm{~kg} / \mathrm{j})$ entre les $P V$ de 300 et $500 \mathrm{~kg}$. On observe que la différence de vitesse de croissance a un effet sur le poids de lipides final plus important chez les Pie-noirs (89 vs $69 \mathrm{~kg}$ ) que chez les Charolais (50 vs $41 \mathrm{~kg}$ ).

Fox DG, Black JR (1984) J Anim Sci 58, 725739

Geay Y, Robelin J, Béranger C (1976) Ann Zootech 25, 287-298

Geay Y, Micol D, Robelin J, Berge P, Malterre C (1987) Bull Tech CRZV Theix INRA 70, 173183

Hoon Song, Dinckel CA (1978) J Anim Sci 47, 70-81

Robelin J (1979) Ann Zootech 28, 209-218

Robelin J (1986) Thèse d'état, Université de Clermont II ( $n^{\circ}$ E268)

Robelin J, Daenicke R (1980) Ann Zootech 29, 99-119

Tableau I. Influence de la vitesse de croissance $(0,7 \mathrm{vs} 1,1 \mathrm{~kg} / \mathrm{j})$ entre 300 et $500 \mathrm{~kg}$, sur le poids $(\mathrm{kg})$ des lipides et des protéines corporelles des bovins mâles Pie-noirs et Charolais.

\begin{tabular}{|c|c|c|c|c|c|c|}
\hline \multirow[t]{3}{*}{ Race } & \multirow{2}{*}{\multicolumn{2}{|c|}{$\begin{array}{l}\text { Etat initial: } \\
\mathrm{PV}=300 \mathrm{~kg}\end{array}$}} & \multicolumn{4}{|c|}{ Etat final: $\mathrm{PV}=500 \mathrm{~kg}$} \\
\hline & & & \multicolumn{2}{|c|}{$d P V=0,7$} & \multicolumn{2}{|c|}{$d P V=1,1$} \\
\hline & LIP & PROT & LIP & PROT & LIP & PROT \\
\hline $\begin{array}{l}\text { Pie-noirs } \\
\text { Charolais }\end{array}$ & $\begin{array}{l}28 \\
20\end{array}$ & $\begin{array}{l}48 \\
49\end{array}$ & $\begin{array}{l}69 \\
41\end{array}$ & $\begin{array}{l}81 \\
89\end{array}$ & $\begin{array}{l}89 \\
50\end{array}$ & $\begin{array}{l}76 \\
86\end{array}$ \\
\hline
\end{tabular}

